\title{
A Model for Conjoint Shape Memory and Pseudo-Elastic Effects during Martensitic Transformation
}

\author{
K. Boubaker \\ Unité de Physique des Dispositifs à Semi-Conducteurs, Faculté des Sciences de Tunis, Tunis El Manar University, 2092 Tunis, Tunisia \\ Correspondence should be addressed to K. Boubaker, mmbb11112000@yahoo.fr \\ Received 9 August 2012; Accepted 30 August 2012 \\ Academic Editors: Y.-D. Wang and Y. Xu \\ Copyright $\odot 2012$ K. Boubaker. This is an open access article distributed under the Creative Commons Attribution License, which \\ permits unrestricted use, distribution, and reproduction in any medium, provided the original work is properly cited. \\ Shape memory alloys (SMA) are metals which can restore their initial shape after having been subjected to a deformation. \\ They exhibit in general both nonlinear shape memory and pseudoelastic effects. In this paper, shape memory alloy (SMA) \\ and its constitutive model with an empirical kinetics equation are investigated. A new formulation to the martensite fraction- \\ dependent Young modulus has been adopted and the plastic deformation was taken into account. To simulate the variations, a \\ one-dimensional constitutive model was constructed based on the uniaxial tension features.
}

\section{Introduction}

Recently, smart metals and alloys have been extensively used in several metallurgical applications, due to their great potential in updated structures and design [1-10]. Among these materials, shape memory alloys (SMA) have attracted more attention, due to their ability to develop extremely large recoverable strains and great forces in the field of biomedical, metallurgy, aerospace, and civil structures [5-10]. In SMA matrices, pseudoplastic effect creates different stress strain behavior resulting in a stress strain curve which lies on the curve produced by the initial linear elastic response during loading. Consecutive and continued unloading may produce linear elastic behavior that eventually returns the structure to the zero stress strain state.

In the present work, an attempt is made to model typical martensitic transformations occurring in shape memory alloys, taking into account pseudoplasticity patterns. In this martensitic transformation, austenite undergoes transformation to form different variants of martensite under a controlled mechanical loading. The formation of martensite in the material is monitored through the coexistence of the initial austenite phases and martensite inside periodic units. Solutions for the implemented governing equations are obtained numerically via explicit numerical protocols and compared to some records presented in the recent related literature [11-18].

\section{Model Patterns}

2.1. Governing Equations. The studied system is a monodimensional rod subjected to axial solicitation (Figure 1). The phase transformations in this considered structure occur by nucleation and growth of platelet inclusions perpendicular to $x$-axis (Figure 1). In this configuration, elastic modulus local expression can be obtained considering the medium as a succession of austenite-martensite periodic units (Figure 1).

The main assumptions of the present model consists of setting one scalar internal variable $\eta$ which represents the martensite fraction, along with linear kinetic rules in terms of the uniaxial stress $T$.

Young modulus $E$ relative to the periodic unit can be computed via the elongation $(x)$ of the periodic cell and the local strain $\varepsilon$ :

$$
\begin{gathered}
x=\varepsilon w=x_{A}+x_{M}=\varepsilon_{A} w_{A}+\varepsilon_{M} w_{M}, \\
\varepsilon=\varepsilon_{A} \frac{w_{A}}{w}+\varepsilon_{M} \frac{w_{M}}{w},
\end{gathered}
$$

where $w, w_{A}$, and $w_{M}$ represent the total length of the periodic cell, the length of the austenite, and martensite fractions, respectively, (Figure 1). Subscripts $A$ and $M$ indicate austenite and martensite. 



FIgURE 1: The studied model.

Since the martensite fraction $\eta$ is defined as

$$
\eta=\frac{w_{M}}{w}
$$

it gives

$$
\varepsilon=\varepsilon_{A}(1-\eta)+\varepsilon_{M} \eta
$$

The $\eta$-dependent boundary condition concerning Young modulus $E(\eta)$ are:

$$
\begin{aligned}
& \left.E(\eta)\right|_{\eta=0}=E_{A}, \\
& \left.E(\eta)\right|_{\eta=1}=E_{M} .
\end{aligned}
$$

The expression of $E(\eta)$ has been discussed by several authors. Tanaka-Mori $[19,20]$ scheme proposed the expression:

$$
\begin{gathered}
E(\eta)=\frac{E_{A} E_{M}}{2}\left[\frac{(1-\eta)+\eta \Pi^{M A}}{E_{M}(1-\eta)+E_{A} \eta \Pi^{M A}}\right. \\
\left.\quad+\frac{(1-\eta) \Pi^{A M}+\eta}{E_{M}(1-\eta) \Pi^{A M}+E_{A} \eta}\right], \\
\Pi^{M A}=\frac{2 E_{M}}{E_{A}+E_{M}}, \\
\Pi^{A M}=\frac{2 E_{A}}{E_{A}+E_{M}},
\end{gathered}
$$

whereas Voigt [21-25] proposed a simple linear model:

$$
E(\eta)=E_{A}(1-\eta)+E_{M}(\eta)
$$

Both expressions verify the imposed condition for Young modulus, but present the disadvantage of lack of control on the first derivatives of $E(\eta)$, which are either sophisticated (Tanaka-Mori scheme $[19,20]$ ) or constant (Voigt [21-25]).
As per the model of derived by Tanaka and Nagaki [26], the main equation deduced from the first and second laws of thermodynamics can be formulated as

$$
T(\varepsilon, \eta)=\rho \frac{\partial \varphi(\varepsilon, \eta)}{\partial \varepsilon}
$$

with

$$
\begin{aligned}
& T: \text { Second Piola-Kirchhoff stress, } \\
& \rho \text { : Density, } \\
& \varphi \text { : Helmholtz free energy, } \\
& \varepsilon: \text { Green strain, } \\
& \eta: \text { Martensite fraction. }
\end{aligned}
$$

In the case of pure mechanical constraint, and taking into accounts the presumptions of Chen et al. [27], the main equation becomes

$$
\begin{gathered}
T(\varepsilon, \eta)-T_{0}=\rho \frac{\partial^{2} \varphi}{\partial \varepsilon^{2}}\left(\varepsilon-\varepsilon_{o}\right)+\rho \frac{\partial^{2} \varphi}{\partial \varepsilon \partial \eta}\left(\eta-\eta_{0}\right) \\
E(\eta)=\rho \frac{\partial^{2} \varphi}{\partial \varepsilon^{2}}
\end{gathered}
$$

where $E(\eta)$ is Young modulus and $T_{0} \varepsilon_{o}$ and $\eta_{0}$ refer to initial condition for stress, strain and martensite fraction, respectively.

In the actual model, the transformation tensor $\rho\left(\partial^{2} \varphi /\right.$ $\partial \varepsilon \partial \eta)$ is considered as constant, while the Young modulus $E(\eta)$ is expressed as

$$
E(\eta)=E_{M}+\frac{E_{M}-E_{A}}{N_{0}} \sum_{k=1}^{N_{0}} \xi_{k} \times B_{4 k}\left(\eta \times r_{k}\right)
$$

where $B_{4 k}$ are the $4 k$-order Boubaker polynomials [28-44], $r_{k}$ are $B_{4 k}$ minimal positive roots, $N_{0}$ is a prefixed integer, and $\left.\xi_{k}\right|_{k=1, \ldots, N_{0}}$ are unknown pondering real coefficients.

This expression refers to the Boubaker polynomials expansion scheme (BPES) [29-31]. This scheme is a resolution protocol which has been successfully applied to several applied-physics and mathematics problems. The BPES protocol ensures the validity of the related boundary conditions regardless of main equation features. The BPES is mainly based on Boubaker polynomials first derivatives properties:

$$
\begin{gathered}
\left.\sum_{q=1}^{N} B_{4 q}(x)\right|_{x=0}=-2 N \neq 0, \\
\left.\sum_{q=1}^{N} B_{4 q}(x)\right|_{x=r_{q}}=0,
\end{gathered}
$$




$$
\begin{gathered}
\left.\sum_{q=1}^{N} \frac{d B_{4 q}(x)}{d x}\right|_{x=0}=0, \\
\left.\sum_{q=1}^{N} \frac{d B_{4 q}(x)}{d x}\right|_{x=r_{q}}=\sum_{q=1}^{N} H_{q},
\end{gathered}
$$

with $H_{n}=B_{4 n}^{\prime}\left(r_{n}\right)=\left(\frac{4 r_{n}\left[2-r_{n}^{2}\right] \times \sum_{q=1}^{n} B_{4 q}^{2}\left(r_{n}\right)}{B_{4(n+1)}\left(r_{n}\right)}+4 r_{n}^{3}\right)$.

Several solutions have been proposed through the BPES in many fields such as numerical analysis [28-31], theoretical physics [31-34], mathematical algorithms [35], heat transfer [36], homodynamic [37, 38], material characterization [39], fuzzy systems modeling [40-42], and biology [43, 44].

The main advantage of this formulation (9) is the verification of boundary conditions, expressed in (4), in advance to resolution process, along with affording controllable and easy-to-access first derivatives of $E(\eta)$. In fact, thanks to the properties expressed in (10), these conditions are inherently verified.

$$
\begin{gathered}
\left.E(\eta)\right|_{\eta=0}=E_{M}+\frac{E_{M}-E_{A}}{N_{0}} \sum_{k=1}^{N_{0}} \xi_{k} \times(-2)=E_{A}, \\
\left.E(\eta)\right|_{\eta=1}=E_{M}+\frac{E_{M}-E_{A}}{N_{0}} \sum_{k=1}^{N_{0}} \xi_{k} \times(0)=E_{M} .
\end{gathered}
$$

Plots of the $\eta$-dependent Young modulus $E(\eta)$ are gathered in Figure 2, for both actual and referred models [19-25].

2.2. Numerical Results and Discussion. Numerical simulation was achieved for the values of parameters gathered in Table 1 . The system was taken as insulated thermally and pinnedend mechanically. A variable mechanical load $T$, expressed as follows, has been considered [45]

$$
T= \begin{cases}3500 t, & t \in\left[0, \frac{\theta}{6}[,\right. \\ 3500(4-t), & t \in\left[\frac{\theta}{6}, \frac{\theta}{3}[,\right. \\ 0, & t \in\left[\frac{\theta}{3}, \frac{\theta}{2}[,\right. \\ 3500(6-t), & t \in\left[\frac{\theta}{2}, \frac{2 \theta}{3}[,\right. \\ 3500(t-10), & t \in\left[\frac{2 \theta}{3}, \frac{5 \theta}{6}[,\right. \\ 0, & t \in\left[\frac{5 \theta}{6}, \theta[.\right. \\ \theta=12 . & \end{cases}
$$

Consequently, the dynamics were simulated using (7)-(8) with the given load (12) and the BPES-related expression (9). Finite element simulations in simple uniaxial solicitation

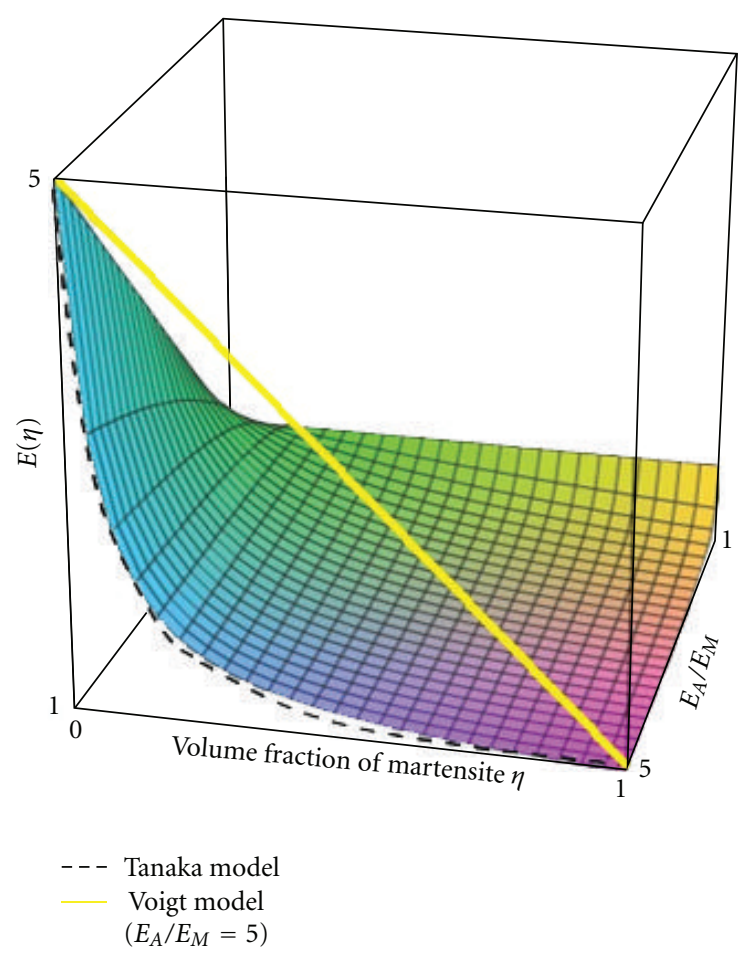

Figure 2: Young modulus $E(\eta)$ versus martensite fraction for different values of $E_{A} / E_{M}$.

TABLE 1: Values of $d_{h k l}$ of relevant parameters.

\begin{tabular}{lcc}
\hline Parameters & Value & Unit \\
\hline$E_{A}$ & $6.010^{5}$ & $\mathrm{MPa}$ \\
$E_{M}$ & $7.510^{3}$ & $\mathrm{MPa}$ \\
Rod diameter & 1.49 & $\mathrm{~mm}$ \\
\hline
\end{tabular}

mode were carried out with the preset load using load gradient and the preset $\eta$-dependent Young modulus expression as input. The resulting stress-strain response was plotted in Figure 3, along with some records from the related literature $[46,47]$.

\section{Discussion and Perspectives}

Numerical stress-strain plots obtained from the actual model confirm that the patterns of hysteresis loop generated in the positive quadrant (Figure 3 ) are in good agreement with the profiles presented by Auricchio [45], Motahari and Ghassemieh [46], and Sayyadi et al. [47].

Strain span shows also a good agreement with the model performed by Motahari and Ghassemieh [46]. The unique divergence lies in the upper zone $(\varepsilon>5 \%)$ and may be attributed to the linear approximation of the $\eta$-dependent Young modulus $E(\eta)$ (Voigt-type) instead of the polynomial form of evolution equations assumed in our study. In this context, it can been stated that Tanaka-Mori $[19,20]$ and Voigt [21-25] models, cannot be efficiently used for predicting the shape memory effect behavior of SMA. This is due to the fact that in the constitutive equations used in those 


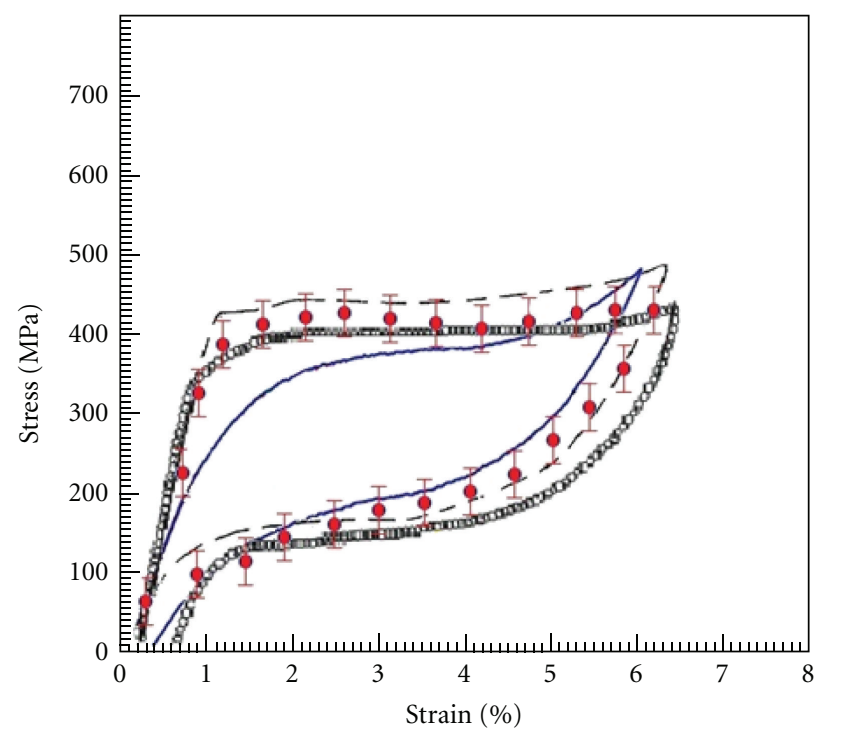

$\begin{array}{lll}\text { - } \text { Auricchio et al., reference [45] } & & \text { Sayyadi et al., reference [47] } \\ \text { mmmm Motahari et al., reference [46] } & \text { I } & \text { Actual model }\end{array}$

FIgURE 3: Solution plots represented in the Strain-Stress plane.

models, the transformational component is proportional to both martensite volume fraction and its derivatives. Indeed, these models, oppositely to the actual one, introduce a prefixed and uncontrollable derivative-dependence.

\section{Conclusion}

In summary, we have implemented constitutive model for shape-memory alloys capable of undergoing austenite to martensite phase transformation using fundamental thermodynamic laws and the principle of martensite fractiondependence. The stress-strain plots obtained from uniaxial monitored load were predicted using the finite-element simulations. A key parameter of the performed model consists of avoiding avoids singularity of the main equilibrium equation during the transition since the derivative of the preset $\eta$ dependent Young modulus expression are controllable. $\eta$ dependent boundary conditions were also inherently taken into account in the model.

Although applied to a particular geometry, the model should be suitable to study other configurations since it was based on a single scalar internal variable: the martensite fraction. This model may be extended to $2 \mathrm{D}$ and $3 \mathrm{D}$, while other possible future developments are the inclusion of permanent inelastic effects, the prediction of coupled thermomechanical behavior, and the nonlinear hardening mechanisms.

\section{References}

[1] G. B. Olson and M. Cohen, "A mechanism for the straininduced nucleation of martensitic transformations," Journal of The Less-Common Metals, vol. 28, no. 1, pp. 107-118, 1972.

[2] T. Angel, "Formation of martensite in austenitic stainless steels: effects of deformation, temperature and composition,"
Journal of The Iron and Steel Institute, vol. 1977, pp. 165-174, 1954.

[3] G. W. Greenwood and R. H. Johnson, "The deformation of metals under stresses during phase transformation," Proceedings of the Royal Society A, vol. 283, no. 1394, pp. 403-422, 1965.

[4] I. Tamura, "Deformation-induced martensitic transformation and transformation-induced plasticity in steels," Journal of Materials Science, vol. 16, no. 5, pp. 245-253, 1982.

[5] J. R. Patel and M. Cohen, "Criterion for the action of applied stress in the martensitic transformation," Acta Metallurgica, vol. 1, no. 5, pp. 531-538, 1953.

[6] R. G. Stringfellow, D. M. Parks, and G. B. Olson, "A constitutive model for transformation plasticity accompanying straininduced martensitic transformations in metastable austenitic steels," Acta Metallurgica et Materialia, vol. 40, no. 7, pp. 17031716, 1992.

[7] Y. Tomita and T. Iwamato, "Constitutive modeling of trip steel and its application to the improvement of mechanical properties," International Journal of Mechanical Sciences, vol. 37, no. 12, pp. 1295-1305, 1995.

[8] J. G. Cook and F. P. Koffyberg, "Solar thermal absorbers employing oxides of Ni and Co," Solar Energy Materials, vol. 10, no. 1, pp. 55-67, 1984.

[9] I. Doghri and A. Ouaar, "Homogenization of two-phase elasto-plastic composite materials and structures study of tangent operators, cyclic plasticity and numerical algorithms," International Journal of Solids and Structures, vol. 40, no. 7, pp. 1681-1712, 2003.

[10] I. Doghri and C. Friebel, "Effective elasto-plastic properties of inclusion-reinforced composites. Study of shape, orientation and cyclic response," Mechanics of Materials, vol. 37, no. 1, pp. 45-68, 2005.

[11] K. Tanaka and T. Mori, "The hardening of crystals by nondeforming particles and fibres," Acta Metallurgica, vol. 18, no. 8, pp. 931-941, 1970.

[12] J. C. Simo and T. J. R. Hughes, Computational Inelasticity, Springer, New York, NY, USA, 1998.

[13] S. Miyazaki, T. Imai, Y. Igo, and K. Otsuka, "Effect of cyclic deformation on the pseudoelasticity characteristics of Ti-Ni alloys," Metallurgical and Materials Transactions A, vol. 17, no. 1, pp. 115-125, 1986.

[14] J. D. Eisenwasser and L. C. Brown, "Pseudoelasticity and the strain-memory effect in Cu-Zn-Sn alloys," Metallurgical and Materials Transactions B, vol. 3, no. 6, pp. 1359-1363, 1972.

[15] S. Miyazaki, S. Kimura, F. Takei, T. Miura, K. Otsuka, and Y. Suzuki, "Shape memory effect and pseudoelasticity in a TiNi single crystal," Scripta Metallurgica, vol. 17, no. 9, pp. 10571062, 1983.

[16] J. Ortin and A. Planes, "Thermodynamic analysis of thermal measurements in thermoelastic martensitic transformations," Acta Metallurgica, vol. 36, no. 8, pp. 1873-1889, 1988.

[17] G. B. Olson and M. Cohen, "Thermoelastic behavior in martensitic transformations," Scripta Metallurgica, vol. 9, no. 11, pp. 1247-1254, 1975.

[18] H. C. Tong and C. M. Wayman, "Thermodynamic considerations of "solid state engines" based on thermoelastic martensitic transformations and the shape memory effect," Metallurgical and Materials Transactions, vol. 6, no. 1, pp. 29-32, 1975.

[19] K. Tanaka and T. Mori, "The hardening of crystals by nondeforming particles and fibres," Acta Metallurgica, vol. 18, no. 8, pp. 931-941, 1970.

[20] K. Tanaka, "Thermomechanical sketch of shape memory effect: one-dimensional tensile behavior," Research Mechanicanical, vol. 18, no. 3, pp. 251-263, 1986. 
[21] J. G. Boyd and D. C. Lagoudas, "Thermomechanical response of shape memory composites," Journal of Intelligent Material Systems and Structures, vol. 5, no. 3, pp. 333-346, 1994.

[22] J. G. Boyd and D. Lagoudas, "A thermodynamical constitutive model for shape memory materials. Part I. The monolithic shape memory alloy," International Journal of Plasticity, vol. 12, no. 6, pp. 805-841, 1996,

[23] J. G. Boyd and D. C. Lagoudas, "A thermodynamical constitutive model for shape memory materials. Part II. The SMA composite material," International Journal of Plasticity, vol. 12, no. 7, pp. 843-873, 1996.

[24] L. C. Brinson and R. Lammering, "Finite element analysis of the behavior of shape memory alloys and their applications," International Journal of Solids and Structures, vol. 4, no. 2, pp. 229-242, 1993.

[25] L. C. Brinson, "One dimensional constitutive behavior of shape memory alloys: thermo-mechanical derivation with non-constant material functions," Journal of Intelligent Material Systems \& Structures, vol. 4, no. 2, pp. 229-242, 1993.

[26] K. Tanaka and S. Nagaki, "A thermomechanical description of materials with internal variables in the process of phase transitions," Ingenieur-Archiv, vol. 51, no. 5, pp. 287-299, 1982.

[27] L. Chen, Constitutive modeling of shape memory alloys [Ph.D. thesis], VPI, Blacksburg, Va, USA, 1990.

[28] A. Milgram, "The stability of the Boubaker polynomials expansion scheme (BPES)-based solution to Lotka-Volterra problem," Journal of Theoretical Biology, vol. 271, no. 1, pp. $157-158,2011$.

[29] J. Ghanouchi, H. Labiadh, and K. Boubaker, "An attempt to solve the heat transfer equation in a model of pyrolysis spray using 4q-order m-boubaker polynomials," International Journal of Heat and Technology, vol. 26, no. 1, pp. 49-53, 2008.

[30] S. Lazzez, K. B. Ben Mahmoud, S. Abroug, F. Saadallah, and M. Amlouk, "A Boubaker polynomials expansion scheme (BPES)related protocol for measuring sprayed thin films thermal characteristics," Current Applied Physics, vol. 9, no. 5, pp. 1129-1133, 2009.

[31] T. Ghrib, K. Boubaker, and M. Bouhafs, "Investigation of thermal diffusivitymicrohardness correlation extended to surfacenitrured steel using Boubaker polynomials expansion," Modern Physics Letters B, vol. 22, no. 29, pp. 2893-2907, 2008.

[32] S. Fridjine, K. B. Ben Mahmoud, M. Amlouk, and M. Bouhafs, "A study of sulfur/selenium substitution effects on physical and mechanical properties of vacuum-grown $\mathrm{ZnS1-xSex}$ compounds using Boubaker polynomials expansion scheme (BPES)," Journal of Alloys and Compounds, vol. 479, no. 1-2, pp. 457-461, 2009.

[33] C. Khélia, K. Boubaker, T. Ben Nasrallah, M. Amlouk, and S. Belgacem, "Morphological and thermal properties of $\beta$-SnS2 sprayed thin films using Boubaker polynomials expansion," Journal of Alloys and Compounds, vol. 477, no. 1-2, pp. 461467, 2009.

[34] M. Dada, O. B. Awojoyogbe, and K. Boubaker, "Heat transfer spray model: an improved theoretical thermal time-response to uniform layers deposit using Bessel and Boubaker polynomials," Current Applied Physics, vol. 9, no. 3, pp. 622-624, 2009.

[35] S. Tabatabaei, T. Zhao, O. B. Awojoyogbe, and F. O. Moses, "Cut-off cooling velocity profiling inside a keyhole model using the Boubaker polynomials expansion scheme," International Journal of Heat and Mass Transfer, vol. 45, no. 10, pp. 1247-1251, 2009.

[36] A. Belhadj, J. Bessrour, M. Bouhafs, and L. Barrallier, "Experimental and theoretical cooling velocity profile inside laser welded metals using keyhole approximation and Boubaker polynomials expansion," Journal of Thermal Analysis and Calorimetry, vol. 97, no. 3, pp. 911-915, 2009.

[37] A. Belhadj, O. F. Onyango, and N. Rozibaeva, "Boubaker polynomials expansion scheme-related heat transfer investigation inside keyhole model," Journal of Thermophysics and Heat Transfer, vol. 23, no. 3, pp. 639-640, 2009.

[38] P. Barry and A. Hennessy, "Meixner-type results for Riordan arrays and associated integer sequences," Journal of Integer Sequences, vol. 13, no. 9, pp. 1-34, 2010.

[39] M. Agida and A. S. Kumar, "A Boubaker polynomials expansion scheme solution to random Love's equation in the case of a rational Kernel," Electronic Journal of Theoretical Physics, vol. 7, no. 24, pp. 319-326, 2010.

[40] A. Yildirim, S. T. Mohyud-Din, and D. H. Zhang, "Analytical solutions to the pulsed Klein-Gordon equation using Modified Variational Iteration Method (MVIM) and Boubaker Polynomials Expansion Scheme (BPES)," Computers and Mathematics with Applications, vol. 59, no. 8, pp. 2473-2477, 2010.

[41] A. S. Kumar, "An analytical solution to applied mathematicsrelated Love's equation using the Boubaker polynomials expansion scheme," Journal of the Franklin Institute, vol. 347, no. 9, pp. 1755-1761, 2010.

[42] M. Benhaliliba, C. E. Benouis, K. Boubaker, M. Amlouk, and A. Amlouk, "The Amlouk-boubaker optothermal expansivity wab in," in The Book: Solar Cells-New Aspects and Solutions, L. A. Kosyachenko, Ed., pp. 27-41, 2011.

[43] H. Rahmanov, "A solution to the non linear korteweg-de-vries equation in the particular case dispersion-adsorption problem in porous media using the spectral Boubaker polynomials expansion scheme (BPES)," Studies in Nonlinear Sciences, vol. 2, no. 1, pp. 46-49, 2011.

[44] C. Khélia, K. Boubaker, and M. Amlouk, "Boubaker polynomials expansion scheme (BPES)-related optical properties of $\beta$-sns2 sprayed layers," Fizika, vol. 18, no. 2, pp. 81-88, 2009.

[45] F. Auricchio, Shape-memory alloys: applications, micromechanics, macro-modelling and numerical simulations [Ph.D. thesis], Department of Civil Engineering, University of California at Berkeley, Berkeley, Calif, USA, 1995.

[46] S. A. Motahari and M. Ghassemieh, "Multilinear onedimensional shape memory material model for use in structural engineering applications," Engineering Structures, vol. 29, no. 6, pp. 904-913, 2007.

[47] H. Sayyadi, M. R. Zakerzadeh, and H. Salehi, "A comparative analysis of some one-dimensional shape memory alloy constitutive models based on experimental tests," Scientia Iranica $B$, vol. 19, no. 2, pp. 249-257, 2012. 

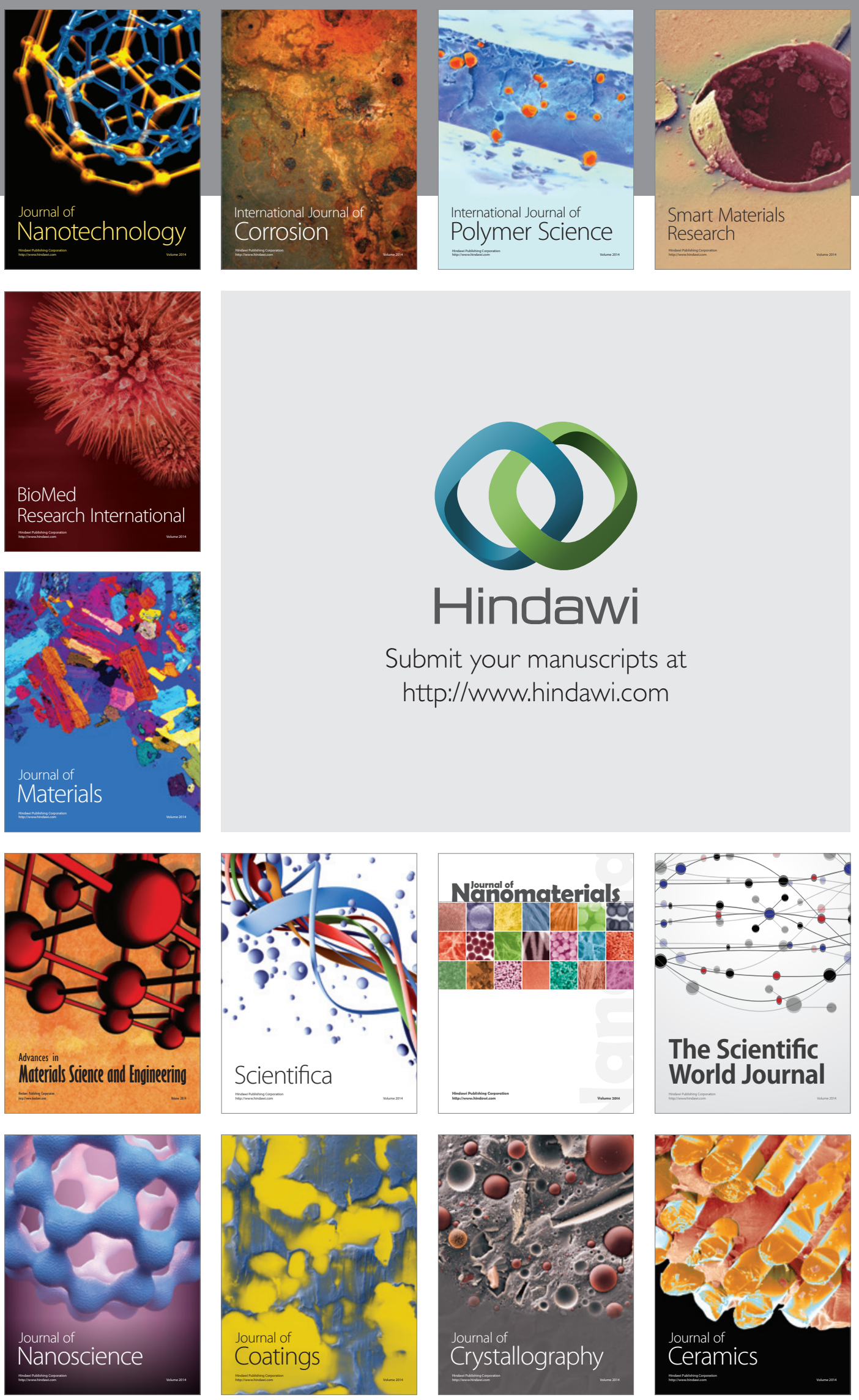

The Scientific World Journal

Submit your manuscripts at

http://www.hindawi.com

\section{World Journal}

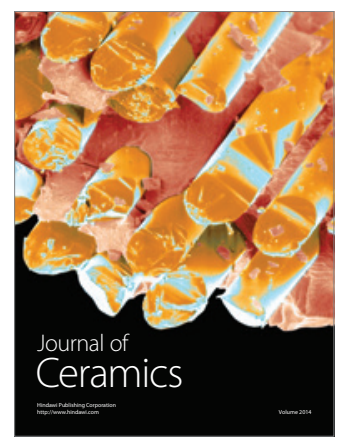

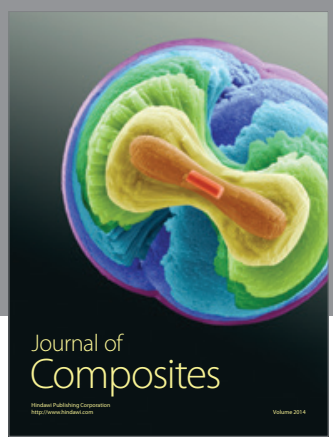
